\title{
Stimulant use still going strong
}

\author{
REVISITING... MISUSE OF AMPHETAMINES AND RELATED DRUGS \\ Nicholas Seivewright, Charles McMahon \& Paul Egleston
}

\begin{abstract}
Amphetamines, cocaine and methylenedioxymethamphetamine (MDMA, 'ecstasy') have been prominent on the UK drugs scene over the past decade. Much cocaine is now in the form of 'crack', which produces particularly acute versions of well-known complications including paranoid psychosis, mood disorders and cardiovascular problems. Ecstasy has additional hallucinogenic properties, and the slightly different range of psychiatric effects can be long-lasting. Assessment for stimulant misuse should include drug screening more than is currently common in general settings. Management comprises psychosocial (particularly behavioural counselling) and pharmacological approaches. A wide range of dopaminergic and other medications have been studied in cocaine misuse, and specialised substitute prescribing may be appropriate for heavy amphetamine injecting. There has been recent focus on problems of dual diagnosis, with particular strategies required to address stimulant misuse by people with severe mental illnesses.
\end{abstract}

This article continues a series revisiting early contributions to APT (see also Cowen, 2005; Edwards, 2005; Gournay, 2005; Mortimer, 2005, this issue). The original article is available on our website (http://apt.rcpsych.org), as a data supplement to the online version of the present article.

In this article we revisit a subject that we reviewed almost a decade ago - the misuse of the most common illicit stimulant drugs (Seivewright \& McMahon, 1996). In the drugs field changes can certainly be expected in that length of time, particularly with substances linked to the so-called recreational drugs scene where fashions and differences in usage come and go. Since the 1960s, when hallucinogens came into fashion, there has been prominence successively of barbiturates, pharmaceutical opioids and then smokable powdered heroin. In the decade leading up to our last review the widespread taking of methylenedioxymethamphetamine (MDMA, 'ecstasy') had emerged, and the long-established use of cocaine had increasingly transferred to its more potent 'crack' form. In 1996 we mainly considered four substances - amphetamine, powdered cocaine hydrochloride, crystalline 'crack' cocaine and MDMA - and since then all have remained highly prevalent on the drugs scene, with amphetamine and MDMA generally found in surveys to be the two most common drugs of misuse in the UK after cannabis.

Table 1 is an updated version of a table that appeared in our earlier review. It shows how the main stimulant drugs are used, approximate current street prices and their classification under the Misuse of Drugs Act 1971, which dictates the severity of penalties for supplying or possession.

So what is different in assessing the problems caused by these drugs ten years on? Gratifyingly, there has been increasing recognition that people heavily injecting amphetamine require just as much attention as the opiate-dependent individuals who have typically predominated in drug services. Consequently, many agencies are devising protocols for stimulant users to run alongside their established methadone and other programmes. The most important psychosocial and pharmacological approaches, which we describe below, depend for their formal evidence base on studies conducted mostly on cocaine users and they have varying applicability to stimulants in general. The tendency for drugs agencies to solely deploy treatments for opiate dependence has been further challenged by the ongoing trends towards polydrug use, with additional cocaine use by opioid substitution

Nicholas Seivewright was senior lecturer in drug dependence in Manchester before moving to his present post as consultant psychiatrist in substance misuse with Sheffield Care NHS Trust (Norfolk House, 4 Norfolk Street, Sheffield S1 2JB, UK. Tel: 0114 271 6802; fax: 0114271 6814; e-mail: amy.robinson@sct.nhs.uk). His research interests include personality disorder, dual diagnosis and pharmacological treatments in addictions, and he has written a practically oriented textbook on drug misuse treatment. Charles McMahon was specialist registrar in substance misuse in Sheffield before taking up his post as consultant psychiatrist in addictions, for NHS Argyll and Clyde. His interests include the treatment of non-opiate drug misuse and psychiatric comorbidity, and managing clinical services. Paul Egleston is a specialist registrar in general psychiatry in Sheffield and was recently an expert contributor to an evidence-based review of drug dependence treatment. He is involved in neuroimaging research and has a career interest in forensic psychiatry. 
Table 1 The main stimulant drugs of misuse

\begin{tabular}{|c|c|c|c|c|}
\hline Drug & Description & $\begin{array}{l}\text { Routes of } \\
\text { administration }\end{array}$ & $\begin{array}{l}\text { Street price, } \\
\text { current }\end{array}$ & $\begin{array}{l}\text { Misuse of Drugs } \\
\text { Act classification }\end{array}$ \\
\hline Amphetamine & $\begin{array}{l}\text { Usually light-coloured powder, low } \\
\text { purity (roughly } 10-20 \% \text { ). Also much } \\
\text { stronger 'base', a moist paste, and } \\
\text { some pharmaceutical preparations }\end{array}$ & $\begin{array}{l}\text { Swallowed, snorted, } \\
\text { intravenous }\end{array}$ & $\begin{array}{l}£ 10-15 \\
\text { per gram }\end{array}$ & $\begin{array}{l}B \text { (A if prepared } \\
\text { for injection) }\end{array}$ \\
\hline $\begin{array}{l}\text { Cocaine } \\
\text { hydrochloride }\end{array}$ & $\begin{array}{l}\text { White powder, moderate purity } \\
\text { (up to } 50 \% \text { ) }\end{array}$ & Snorted, intravenous & $\begin{array}{l}£ 40-60 \\
\text { per gram }\end{array}$ & A \\
\hline Crack cocaine & Crystalline 'rocks' & Smoked, intravenous & $\begin{array}{l}£ 10-20 \text { per } \\
150 \mathrm{mg} \text { rock }\end{array}$ & A \\
\hline MDMA & $\begin{array}{l}\text { Various manufactured tablets, often } \\
\text { with characteristic motifs }\end{array}$ & Swallowed & $\begin{array}{l}£ 5-10 \text { per } \\
100-120 \mathrm{mg} \\
\text { tablet }\end{array}$ & A \\
\hline
\end{tabular}

patients currently particularly problematic (Gossop et al, 2002). There has been no reversal, at least in the UK, of the rise in crack cocaine use, as distinct from intranasal use of the hydrochloride form. Particularly acute physical and psychological complications occur in crack users, who are often from marginalised social groups. The effects of MDMA are somewhat different in nature and there has been more progress in delineating the psychiatric problems that users of this drug can develop, as well as the medical complications, which can sometimes be fatal.

Finally by way of overview, we would reiterate the relevance of stimulant misuse for the majority of psychiatrists, not just those working in substance misuse services. It is well known that, through their common action in enhancing central transmission of catecholamines, amphetamine and cocaine can produce psychoses resembling schizophrenia, which in acute circumstances are most likely to be treated in general psychiatric settings. Furthermore, as people with severe mental illness are predominantly managed in the community very large numbers take drugs, including stimulants. This creates additional problems, with such 'dual diagnosis' cases frequently requiring combined service provision and special attention to issues such as adherence to psychiatric medication regimens (Swofford et al, 2000; Weaver et al, 2003). Careful assessment of the relative contributions of these drugs and of the underlying illness to observed clinical states is often necessary. Clinicians should be wary of the oversimplistic application of the term 'drug-induced psychosis', and be prepared to include drug misusers within systematic management schemes such as the UK's care programme approach (CPA) if their condition merits it. The need to 'mainstream' the management of people with dual diagnosis, with ready use of CPA and treatment within general mental health services wherever possible, has been formalised as policy in good practice guidelines from the Department of Health (2002).

\section{Usage}

\section{Amphetamine}

Most use of this drug is in the form of the relatively impure powder commonly known as 'speed' or 'whizz'. The majority of users take the drug orally, for instance by wrapping some in a cigarette paper and swallowing ('bombing'), or putting it in a drink. In the past decade there has been increased use of the 'base' preparation, which is moist to the touch and generally much more potent in its effects, including causing psychotic reactions. A version seen in some countries, but not significantly in the UK, is methamphetamine ('ice'), although confusingly some laboratory analyses label MDMA as methamphetamine. Pharmaceutical amphetamines are only rarely encountered in the street-drugs scene, although this may change if there is a significant increase in the very specialised area of substitute prescribing for some heavily injecting users (see below).

\section{Cocaine}

Cocaine hydrochloride is more expensive than other illicit drugs and use by snorting has sometimes been linked with executive lifestyles. This same powdered preparation has also long been injected by polydrug users. The crack form is generally more potent in terms of effects and withdrawal features. Rapid rises in blood levels, and hence a very quick high, occur after smoking crack in various kinds of pipe or container, but committed injectors use their preferred route. 


\section{MDMA (ecstasy)}

Ecstasy was inextricably associated with the rise of the rave dance culture across Europe and elsewhere, and the links with 'clubbing' generally continue. Amphetamine and lysergic acid diethylamide (LSD) are frequently used in the same club scenes, and large numbers of individuals take these as part of perceived normalised socialising, with a mainly low likelihood of progression to dependent or injected drug use. Ecstasy tablets are made in illicit laboratories and may be stamped with various motifs, and users seek out their own favoured type. Periodically there have been additional concerns that totally unrelated substances such as the anaesthetic agent ketamine are being passed off as MDMA. The similar compounds 3,4-methylenedioxyethylamphetamine (MDEA) and 3,4-methylenedioxyamphetamine (MDA) are also sometimes encountered.

Surveys tend to show levels of usage of any stimulant drug in the UK as small, single-figure percentages of the general population, but in young people usage can rise to $5-10 \%$ or even more. There is an impression that the MDMA phenomenon is on the wane, and this was demonstrated in a survey of older school students by Plant \& Miller (2000). They found that lifetime use by boys had decreased from 9 to $3 \%$ between 1995 and 1999, and by girls from 7 to $3 \%$. The issue of sampling in drug misuse research is crucial, as illustrated in one comprehensive comparison of MDMA use between a general population sample, regular users and 'purposive' samples of high-using and minority groups (Topp et al, 2004). Patterns of usage were well demonstrated in their study: notably, no individuals in any group were taking MDMA daily, and alcohol, cannabis and amphetamines were the most common additionally taken substances.

\section{Clinical features}

The clinical features of stimulant usage were described in our previous article (Seivewright \& McMahon, 1996) and are generally well established. The main mechanism that produces the stimulant action and related effects comprises increased presynaptic release and inhibition of reuptake of catecholamines, with a direct action on dopaminergic terminals and effects in the common 'reward pathway'. The characteristic features, which we have classified into early, late and withdrawal effects, are indicated in Box 1.

The use of the term 'withdrawal' can be questioned by purists as the stimulants are not truly addictive, at least in the physical sense in which heroin, benzodiazepines or alcohol are. Nevertheless, there is a clear distinction between early effects, which are mainly the desired ones, the features that supervene, which are more adverse in nature and usually lead the individual to end the episode of usage, and finally the stage that users refer to as the come-down or crash. After the effects of stimulation, this withdrawal stage partly constitutes rebound (i.e. opposite) symptoms of oversleeping, overeating and depressed mood, but there may also be agitation, irritability and general distress, which commonly lead to other substance use in attempts at alleviation. Stimulant users often counter withdrawal with alcohol, tranquillisers or cannabis, or heroin may be used in this way with dependence developing. Cocaine and alcohol are also frequently used in a straightforward combination, with individuals presenting with combined dependence.

Cocaine is shorter-acting than amphetamine and each instance of use tends to be over more quickly, in sessions lasting perhaps a few hours. Many committed stimulant users, however, take their drug over 2-3 days, keeping awake during the nights and eating little. They readily use the term paranoia fairly accurately for the states of ideas of reference, other paranoid ideation and actual delusions that can develop after that length of time, usually recognising that they should stop the episode until they feel more normal again. This usually averts a fully developed paranoid psychosis, although this can, of course, occur especially after prolonged and heavy use of amphetamine or cocaine.

In addition to the general stimulant features, MDMA also has partly hallucinogenic, or 'psychedelic', effects resembling those of LSD. These include visual illusions, general enhancement of sensory perceptions and states of altered consciousness, which along with a feeling of emotional closeness to other people are basically the desired effects in typical usage. Common physical effects include tachycardia, dry mouth, dilated pupils, facial muscle stiffness and paraesthesia. Negative effects such as sickness, confusion, mood disturbances and ataxia are also experienced by many users (Handy et al, 1998).

\section{Box 1 Effects of stimulant drugs}

\begin{tabular}{|c|c|}
\hline Early & $\begin{array}{l}\text { Increased energy, elation, reduced } \\
\text { appetite }\end{array}$ \\
\hline Late & $\begin{array}{l}\text { Overactivity, insomnia, confusion, } \\
\text { paranoia }\end{array}$ \\
\hline Withdrawal & $\begin{array}{l}\text { Depression, irritability, agitation, } \\
\text { craving, hyperphagia, sleep dis- } \\
\text { turbance (hypersomnia, night- } \\
\text { mares) }\end{array}$ \\
\hline
\end{tabular}


Box 2 Possible complications of stimulant misuse

Medical

- Cardiovascular: hypertension, arrhythmias, myocardial infarction, cerebrovascular accident

- Infective: abscesses, hepatitis, septicaemia, HIV

- Obstetric: reduced foetal growth, miscarriage, placental abruption, premature labour

- Other: hyperthermia, weight loss, dental problems, epilepsy

Psychiatric

- Anxiety

- Depression

- Aggressive behaviour

- Confusional state

- Paranoid psychosis

\section{Complications}

The range of clinical features, including adverse and withdrawal effects, merge into more definite complications, a classification of which is given in Box 2 . Some complications clearly relate to subgroups of users only, such as injectors or pregnant women; in the latter, stimulants appear to cause higher levels of obstetric complications than are expected from drug misuse in general.

Importantly, because of their primary effect on monoamines the stimulant drugs are relatively likely to produce vascular effects that can lead to myocardial infarction and stroke. High proportions of young people who have died from these conditions in the USA have been found to be cocaine misusers.

Of the infective conditions, the one that has been recognised in the past decade to be of great significance is hepatitis $\mathrm{C}$, which can lead to chronic liver impairment and is commonly found in up to $50 \%$, and sometimes in over $90 \%$, of drug injectors in different areas. Antiviral treatments can be used for the condition, although many units operate policies whereby injecting must have stopped before treatment is provided (Schaefer et al, 2004).

From the listed psychiatric effects it will already have become clear that stimulants frequently produce mood disturbances or confusion. The aggressive behaviour that can undoubtedly occur as a direct effect of drug intoxication needs to be distinguished from the more general antisocial personality features that are common in drug misusers (Bowden-Jones et al, 2004). Paranoid psychosis from amphetamine use has been recognised for decades, with even more acute similar syndromes increasingly occurring from crack cocaine. It seems that dopaminergic overactivity substantially accounts for this schizophrenia-like condition. There is now controversy as to the representativeness of the early reports of amphetamine psychosis, which described only a relatively shortlived condition that resolved if the drug was withdrawn (Curran et al, 2004). The exact relationships between transient psychotic symptoms, more persistent illness and the effects of substance misuse are not fully established, but in practice the misuse of stimulant drugs is highly likely to have a deleterious effect on any individual with a pre-existing psychotic condition. In stimulant psychoses tactile and visual hallucinations probably occur more frequently than in schizophrenia, and there may be hyperactivity and repetitive or compulsive behaviours. If the conditions do become chronic, for instance with ongoing drug use, there is less deterioration in terms of negative symptoms from the drug-related psychoses. One interesting study found higher levels of overt behavioural disturbance in individuals with substance use disorder and psychosis (dual diagnosis), but better preservation of social competence (Penk et al, 2000).

It was known when we last reviewed this subject that MDMA could produce a range of psychiatric after-effects such as anxiety states, depression, flashback experiences, persistent cognitive deficits and occasionally psychoses, and also that the drug could be fatal in isolated cases even after single usage. The literature on psychiatric sequelae continues to be mainly small case series, and the importance of other vulnerability factors and the confounding effects of additional substance use have been emphasised (Soar et al, 2001). Regarding severe physical consequences, it has become increasingly clear that as well as general stimulant adverse effects and other toxicity the most likely fatal syndrome from MDMA includes hyperthermia, rhabdomyolysis, renal failure and disseminated intravascular coagulation (Gowing et al, 2002). Dehydration is thought to contribute to this syndrome as well as the chemical itself, as MDMA is usually used in the context of prolonged dancing in hot crowded environments.

\section{Management}

\section{Assessment}

As in other areas of substance misuse, assessment is mainly through history, examination and relevant investigations. Details must be established of use of the drugs in question, bearing in mind that multiple substance use is common. Additional assessments may be necessary for the various complications. It is 


$\begin{array}{lll}\text { Table } 2 & \text { Testing for misuse of stimulant drugs } \\ \text { Sample } & \begin{array}{l}\text { Detection } \\ \text { period }\end{array} & \text { Remarks } \\ \text { Urine } & \text { 1-3 days } & \begin{array}{l}\text { Usual method; should be } \\ \text { routine where drug use } \\ \text { is possible. Laboratory } \\ \text { testing or instant kits } \\ \text { Specialised laboratory } \\ \text { Oral fluid }\end{array} \quad \text { 1-2 days } \\ & & \begin{array}{l}\text { Useful if there are } \\ \text { compliance or authenticity } \\ \text { problems with urine } \\ \text { Hair }\end{array} \\ & \text { Permanent } & \begin{array}{l}\text { Specialised and relatively } \\ \text { expensive. Examination of } \\ \text { sections gives chronology } \\ \text { of drug usage, e.g. for } \\ \text { medico-legal cases }\end{array}\end{array}$

important to establish a user's personal situation and the context in which they take drugs, as well as making a broad assessment of their motivation to change their behaviour.

The usage history should include amounts, frequency, routes of administration, duration of usage, relevant treatments received and significant drug-free periods. There are few specific physical signs of stimulant usage beyond dilated pupils and overactivity, although needle sites may be seen in injecting users. Physical examination can reveal medical complications, and drug screening often provides essential information, particularly when monitoring whether an individual with psychiatric complications is or is not abstaining from drugs. Managing a drug misuser without urine results is rather like treating a haematology patient without blood counts, and with the generally high levels of current drug use it would seem that there needs to be a greater awareness of testing in general psychiatric settings.

Table 2 summarises the main drug-screening methods and the period after a drug has been taken within which they are effective. Urine sampling is by far the most routine. The process is simple, using a plain bottle and either instant testing with a kit or laboratory analysis. Compliance may be a problem in disturbed individuals, and the recent development of equipment for easily obtaining cheek cell fluid samples provides a method that is often more acceptable. Hair testing is a specialised approach which has the advantage that most substances remain detectable for as long as the hair is present.

\section{Harm reduction}

Even with some recent developments in treatment the clinical management of stimulant misuse is not nearly as effective as that for opiate dependence, where treatments such as methadone and buprenorphine routinely enable substantial reductions in drug usage in the majority of individuals. Partly because there is no equivalent of these established and readily available substitution therapies, stimulant users are far less likely to present to medical services. However, stimulant users need harm reduction advice, which can be provided by community drug teams and specialised non-statutory services. Thus, at the more informal end of the range of management approaches are drugs information, education about health risks, advice on safe practices and the provision of clean injecting equipment.

\section{Psychosocial treatments}

This term is applied to the more systematic counselling and behavioural approaches that are undertaken by nurses, psychologists or social workers in substance misuse or sometimes general psychiatry teams. Often there needs to be some focus on motivation to change, as this is not static and can be enhanced by well-established techniques of motivational interviewing, sometimes allied to other cognitive-behavioural strategies and coping skills training (Rohsenow et al, 2004). In a survey investigating treatments for cocaine use deployed in UK services, the overall category of counselling was by far the most common approach. Detailed follow-up of a subgroup of clients, mainly in residential rehabilitation units, showed good outcomes, with improvements in health and psychological adjustment (Seivewright et al, 2000). A large multicentre study of psychosocial treatments for cocaine dependence in the USA found that individual and group therapy based on treatment manuals compared favourably with some of the more specialised psychotherapeutic techniques (CritsCristoph et al, 1999).

Psychophysiological methods can be used by specialist practitioners, particularly for cocaine misuse, in which there is high arousal to drug-related cues that may be reduced through systematic exposure.

A very different behavioural approach has also been investigated, that of providing rewards such as retail vouchers for demonstrable abstinence from drugs during ongoing treatment. The latest in a series of studies using this 'community reinforcement therapy' with cocaine-dependent individuals from one centre in the USA demonstrates benefits in retention in treatment and social outcomes such as employment, and reduced drug and alcohol use (Higgins et al, 2003). The idea of financially rewarding users to abstain is inevitably controversial, but the results have been impressive in comparison with other treatments. 
A systematic programme comprising cognitivebehavioural therapy, family education, social support groups and individual counselling has also been found in a large multisite study to be beneficial for the treatment of methamphetamine dependence (Rawson et al, 2004), and such methods have broad relevance for managing stimulant misuse in the UK.

\section{Pharmacological treatments}

Both harm reduction and psychosocial treatments for stimulant users are extremely important, since there has been no major progress in demonstrating useful pharmacological treatments. As crack cocaine use escalated in many countries there was great pressure on researchers to find a medication as effective for that drug as methadone is for heroin dependence, but the nature of the dependent states is very different. Research efforts focused on dopaminergic drugs (including bromocriptine and amantadine), serotonergic reuptake inhibitors, other antidepressants, antipsychotics, mood-stabilising medications and even disulfiram, the last mainly to prevent cocaine misuse in individuals who only take the drug after alcohol. Although there have been positive results from uncontrolled studies, notably with desipramine and fluoxetine, the large metaanalyses now conclude that there is no strong evidence for any medication in cocaine dependence treatment (e.g. de Lima et al, 2002). The most common direct usage in practice is of antidepressants to treat definite evidence of a depressive disorder, with other medications as indicated for psychiatric complications.

In UK drug services it is common for patients on methadone also to use cocaine (Gossop et al, 2002), and in such cases there is at least a theoretical advantage in changing the maintenance agent to buprenorphine, as this partial agonist leads to less in the way of combined subjective effects. The use of disulfiram for cocaine addiction has received a good deal of publicity, and it appears that as well as preventing individuals taking cocaine after alcohol the medication may have a more specific effect on features of cocaine use (Carroll et al, 2004).

As we have noted, a substitution approach is not usually considered suitable in cocaine misuse, although there have been trials of both methylphenidate and dexamphetamine. It might indeed be assumed that the potentially destabilising and sometimes psychotic effects of stimulants would simply rule out such therapies, but some specialised units accommodate oral dexamphetamine prescribing for the heaviest and most problematic amphetamine users who otherwise inject the street preparation several times daily. Such prescribing is seen as based on harm reduction principles,
Box 3 Some distinctions within stimulant misuse management

Amphetamines

- Most cases minor, but heavy use merits systematic treatment

- General drug counselling is the mainstay, with limited evidence for antidepressants in withdrawal

- Substitute prescribing sometimes undertaken in specialist services for daily injecting users

Cocaine

- Psychological and physical complications often acute, particularly with crack

- Very large number of medications trialled in studies but few positive results

- Specialised psychological treatments developed, e.g. cue exposure and behavioural reinforcement

$M D M A$

- Elective presentation for treatment is rare

- No special programmes available

- Likely area of involvement is advising on psychiatric after-effects

and some reasonable results have been achieved (Grabowski et al, 2004). In practical terms many UK drug services operate a three-tier approach for amphetamine users, offering counselling alone for most cases, some use of symptomatic medications in individuals who find it particularly hard to overcome the period of withdrawal, and limited dexamphetamine prescribing in the most severe cases (Seivewright, 2000: pp. 128-132).

Some of the same management approaches may be relevant for MDMA users, but these individuals present relatively rarely to drug treatment services, typically seeing their drug-taking as 'recreational' despite the various adverse effects.

Although we have considered the treatment of stimulant misuse collectively there are some important differences in emphasis between the substances, and these are summarised in Box 3 .

\section{Other}

Complementary therapies are frequently deployed for stimulant users, particularly in non-clinical services, with acupuncture the most established method (Seivewright et al, 2000). There is little systematic evidence for effectiveness of the methods overall, nor for the very different experimental approaches of enhancement of drug excretion and antibody formation from cocaine vaccination. 


\section{Treatments for psychiatric complications}

As a basic principle, anxiety states, depression, paranoid psychosis, confusional states and other behavioural disturbances produced by stimulants should be managed in much the same way as such conditions of other aetiology. The serotonergic antidepressants are sometimes favoured because they may reduce drug craving, although there is equal theoretical rationale for compounds such as desipramine, which act more on monoamines. A pragmatic consideration is the preferable avoidance of benzodiazepines in managing drug users, because of the potential for misuse. The issue of stimulant psychosis has received careful consideration in a review by Curran et al (2004), which addresses whether a kind of sensitisation occurs to even limited drug exposure which might indicate ongoing antipsychotic drug treatment. The examination of many studies suggests that this may sometimes be necessary despite the usual view that amphetamine psychosis is short-lived, and it is also concluded that stimulant misuse has the potential to worsen symptoms in individuals with severe mental illness even when they are adhering to antipsychotic drug regimens.

\section{Issues of dual diagnosis}

We have reiterated that individuals who misuse stimulants may become psychotic as a direct effect and that many patients with illnesses such as schizophrenia additionally use various substances. The much-used term 'drug-induced psychosis' actually covers a range of phenomena, including the classic paranoid states, syndromes of a more organic nature with illusions and visual hallucinations produced by LSD or MDMA, briefer flashback experiences and/or worsening or alteration of pre-existing psychotic conditions (Poole \& Brabbins, 1996). As the management of drug-induced states is basically along standard symptomatic lines, much of the recent specialised literature addresses the clinical approaches necessary for people with severe mental illness who develop substance misuse, given the implications that such dual diagnosis has for severity of psychiatric disturbance, adherence to treatments and overall prognosis (Seivewright et al, 2004).

Some units in the USA have developed ultraspecialised programmes solely for dual diagnosis patients, often with high staffing levels and relatively small case-loads. The development of such centres in the UK seems unlikely, in light of the general policy preference to build on existing links between community mental health teams, substance misuse services and primary care, and to treat dual diagnosis cases in the mainstream as far as possible.
Nevertheless, clinical guidance from experienced units is very useful in identifying the principles often necessary in treating this group (Drake \& Mueser, 2000). These include assertive outreach if adherence is a problem, close monitoring, integration between services and the ability to tackle very practical aspects such as finances and accommodation as well as deploying formal therapies.

A common difficulty in practice is that people with distressing psychiatric symptoms can subjectively feel that they gain relief from taking drugs, whether they be sedatives to subdue hallucinations or stimulants to counter lethargy, depression and negative symptoms. Consequently, careful advice on drug-related harms tailored to the requirements of this population needs to be provided. In general it can be expected that stimulant users presenting in psychiatric services who have not actually requested treatment for drug misuse will be suitable for motivational approaches in the first instance rather than the various forms of cognitive therapy.

A particularly comprehensive recent study of dual diagnosis in the UK (Weaver et al, 2003) included both a survey of all the individuals on the case-load of keyworkers in community mental health and substance misuse services in three cities on a given date, and thorough formalised assessments of a random subsample. Response rates were high and the research confirmed an impression that services currently mainly manage individuals with severe disorders, with $75 \%$ of the mental health interview sample having psychosis. Thirty-one per cent of that sample currently used illicit drugs (mainly stimulants, cannabis, tranquillisers and heroin), while of the interviewed drug misuse patients over $70 \%$ had significant additional psychiatric illness. A measure was included of risk factors that would indicate referral for CPA, and in general there were fewer contacts with reciprocal services for dual diagnosis patients than appeared necessary on clinical grounds.

Weaver et al's study reinforces the need for more liaison and joint working, and it is to be hoped that education on the various manifestations of substance misuse problems and the management of relevant psychiatric conditions will help enable that.

\section{References and related articles}

Bowden-Jones, O., Iqbal, M. Z., Tyrer, P., et al (2004) Prevalence of personality disorder in alcohol and drug services and associated comorbidity. Addiction, 99, $1306-1314$

Carroll, K. M., Fenton, L. R., Ball, S. A., et al (2004) Efficacy of disulfiram and cognitive behavior therapy in cocaine dependent outpatients: a randomized placebocontrolled trial. Archives of General Psychiatry, 61, 264272.

Cowen, P. J. (2005) New drugs, old problems. Advances in Psychiatric Treatment, 11, 19-27. 
Crits-Cristoph, P., Siqueland, L., Blaine, J., et al (1999) Psychosocial treatments for cocaine dependence. Archives of General Psychiatry, 56, 493-502.

Curran, C., Byrappa, N. \& McBride, A. (2004) Stimulant psychosis: systematic review. British Journal of Psychiatry, 185, 196-204.

de Lima, M. S., de Oliveira Soares, B. G., Reisser, A. A. P. \& Farrell, M. (2002) Pharmacological treatment of cocaine dependence: a systematic review. Addiction, 97, 931-949.

Department of Health (2002) Mental Health Policy Implementation Guide: Dual Diagnosis Good Practice Guide. London: Stationery Office.

Drake, R. E. \& Mueser, K. T. (2000) Psychosocial approaches to dual diagnosis. Schizophrenia Bulletin, 26, 105-118.

Edwards, J. G. (2005) Newer v. older antidepressants in longterm pharmacotherapy. Revisiting... Prevention of relapse and recurrence of depression. Advances in Psychiatric Treatment, 11, 184-194.

Gournay, K. (2005) The changing face of psychiatric nursing. Advances in Psychiatric Treatment, 11, 6-11.

Gowing, L. R., Henry-Edwards, S. M. \& Irvine, R. G. (2002) The health effects of ecstasy. Drug and Alcohol Review, 21, 53-63.

Gossop, M., Marsden, J., Stewart, D., et al (2002) Changes in use of crack after drug misuse treatment: 4-5 year followup results from the National Treatment Outcome Research Study (NTORS). Drug and Alcohol Dependence, 66, 21-28.

Grabowski, J., Shearer, J., Merrill, J., et al (2004) Agonist-like, replacement pharmacotherapy for stimulant abuse and dependence. Addictive Behaviours, 29, 1439-1464.

Handy, C., Pates, R. \& Barrowcliff, A. (1998) Drug use in South Wales: who uses Ecstasy anyway? Journal of Substance Misuse, 3, 82-88.

Higgins, S. T., Sigmon, S. C., Wong, C. J., et al (2003) Community reinforcement therapy for cocaine-dependent outpatients. Archives of General Psychiatry, 60, 1043-1052.

Mortimer, A. (2005) Another triumph of hope over experience? Advances in Psychiatric Treatment, 11, 277-285.

Penk, W. E., Flannery, R. B. Jr, Irvin, E., et al (2000) Characteristics of substance-abusing persons with schizophrenia: the paradox of the dually diagnosed. Journal of Addictive Diseases, 19, 23-30.

Plant, M. \& Miller, P. (2000) Drug use has declined amongst teenagers in United Kingdom. BMJ, 320, 1536-1537.

Poole, R. \& Brabbins, C. (1996) Drug induced psychosis. British Journal of Psychiatry, 168, 135-138.

Rawson, A. R., Marinelli-Casey, P., Anglin, M. D., et al (2004) A multi-site comparison of psychosocial approaches for the treatment of methamphetamine dependence. Addiction, 99, 708-717.

Rohsenow, D. J., Monti, P. M., Martin, R. A., et al (2004) Motivational enhancement and coping skills training for cocaine abusers: effects on substance use outcomes. Addiction, 99, 862-874.

Schaefer, M., Heinz, A. \& Backmund, M. (2004) Treatment of chronic hepatitis $C$ in patients with drug dependence: time to change the rules? Addiction, 99, 1167-1175.

Seivewright, N. (2000) Community Treatment of Drug Misuse: More Than Methadone. Cambridge: Cambridge University Press.

Seivewright, N. \& McMahon, C. (1996) Misuse of amphetamines and related drugs. Advances in Psychiatric Treatment, 2, 211-218

Seivewright, N., Donmall, M., Douglas, J., et al (2000) Cocaine misuse treatment in England. International Journal of Drug Policy, 11, 203-215.

Seivewright, N., Iqbal, M. Z. \& Bourne, H. (2004) Treating patients with comorbidities. In Drug Treatment: What Works? (eds P. Bean \& T. Nemitz), pp. 123-141. London: Routledge.

Soar, K., Turner, J. J. D. \& Parrott, A. C, (2001) Psychiatric disorders in Ecstasy (MDMA) users. A literature review focusing on personal predisposition and drug history. Human Psychopharmacology, 16, 641-645.

Swofford, C. D., Scheller-Gilkey, G., Miller, A. H., et al (2000) Double jeopardy: schizophrenia and substance use. American Journal of Drug and Alcohol Abuse, 26, 343-353.

Topp, L., Barker, B. \& Degenhardt, L. (2004) The external validity of results derived from ecstasy users recruited using purposive sampling strategies. Drug and Alcohol Dependence, 73, 33-40.

Weaver, T., Madden, P., Charles, V., et al (2003) Comorbidity of substance misuse and mental illness in community mental health and substance misuse services. British Journal of Psychiatry, 183, 304-313.

\section{MCQs}

1 Crack cocaine:

a is mainly taken intranasally

b produces more potent effects than cocaine hydrochloride

c may lead to paranoid psychosis if used heavily

$\mathrm{d}$ is avoided by individuals on methadone because of interaction

e enhances central transmission of catecholamines.

2 Characteristic effects of MDMA include:

a visual illusions

b stimulation

c pupillary constriction

d feeling of emotional closeness to other people

e diarrhoea.

3 In screening tests for drugs of misuse:

a sampling of urine and hair are equally routine

b cocaine cannot be detected because of its short half-life

c amphetamines used a day before urine sampling may be identified

$\mathrm{d}$ a positive result from an individual in a psychotic episode confirms drugs as the aetiology

e oral fluid sampling is only used in specialised forensic analysis.

4 Recognised treatments for amphetamine misuse include:

a buprenorphine

b general drug counselling

c motivational interviewing

d substitute prescribing in severe cases

e carbamazepine.

5 In the management of dual diagnosis:

a stimulant use can worsen an existing psychotic condition

b the persistence of paranoid delusions for more than 1 week confirms a diagnosis of schizophrenia

c the care programme approach excludes patients with substance misuse

d cocaine use may impair compliance with psychotropic drug treatment

e assertive outreach is recognised as sometimes required.

\section{MCQ answers}

\begin{tabular}{|c|c|c|c|}
\hline & 2 & 3 & 4 \\
\hline $\mathrm{F}$ & a $\mathrm{T}$ & a $F$ & a $F$ \\
\hline $\mathrm{T}$ & b $\mathrm{T}$ & $b F$ & $\mathrm{~b} T$ \\
\hline $\mathrm{T}$ & C F & c $\mathrm{T}$ & C $\mathrm{T}$ \\
\hline F & $\mathrm{d} T$ & d F & $\mathrm{d} \mathrm{T}$ \\
\hline $\mathrm{T}$ & e $F$ & e F & e F \\
\hline
\end{tabular}

